\title{
Impact of Inertia Distribution on Power System Stability and Operation
}

\author{
Bahman Alinezhad Osbouei, \\ Gareth A. Taylor, \\ Dept. of Electrical Engineering \\ Brunel University London \\ London, United Kingdom \\ bahman.alinezhadosbouei@brunel.ac.uk
}

\author{
Olivier Bronckart ${ }^{1}$, \\ Johan Maricq², \\ ${ }^{1}$ Power System Operations and Security \\ ${ }^{2}$ Innovation and Strategy \\ Elia Group \\ Brussels, Belgium
}

\author{
Martin Bradley, \\ Dispatch Assurance Manager \\ Electricity Operations \\ National Grid \\ London, United Kingdom
}

\begin{abstract}
This paper presents the local inertia phenomenon which is related to the uneven distribution of inertia across a synchronized power system and its influence on the system stability and operation. This study explains that beyond the question of overall system inertia as required at the synchronous area level, the distribution of inertia may have an impact on the system local behaviour and operation particularly in smaller synchronous systems. In this area, some specific and relevant events observed by UK National Grid are simulated on a 36zone GB transmission network reduced model to identify the possible effects due to an uneven distribution of inertia. In addition, in this paper, the accuracy of this model is verified and fine-tuned with the data obtained from real measurements. Based on the obtained results, higher local RoCoF, increasing the inter-area oscillations as well as changes in critical clearing time of tie-lines are the expected effects of local inertia phenomenon.
\end{abstract}

Keywords - Local inertia, Inertia distribution, Rate of Change of Frequency (RoCoF), Inter-area oscillations, Power system stability

\section{INTRODUCTION}

As a part of current EU climate and energy strategy, the proportion of electricity generation obtained from renewable energy resources (RES) should increase up to $55 \%$ in 2050 [1]. Since most of RES are implementing power electronic converters in their interconnecting interface, they are generally interpreted as non-synchronous generation. Following an increasing share of non-synchronous generation and power electronic loads in the power systems and a decreasing share of synchronous units (the latter provides the main part of the system inertia today), the overall system inertia as well as its distribution across the system is reduced and changed respectively [2].

As stated by the system protection $\&$ dynamics sub group of ENTSO-E, "decreased system overall inertia will have a significant impact if the continental EU power system faces a system split". However, based on the recent observations, it is discovered that beyond the question of overall system inertia, the distribution of inertia may have an impact on the local behaviour or operation of the areas with a relatively small inertia in a synchronous power system [3]. In such systems, the activation of RoCoF-based protection in certain locations and inter-area oscillations are some of the possible effects due to an uneven distribution of inertia.

The term Local Inertia phenomenon introduced here denotes to a power system with interconnected synchronized areas but with different inertia at each one (uneven distribution of inertia across the entire system). For such a system, there may exist some large conventional synchronous generators with a high inertia in some areas while there exists significant renewable power generation with low or zero rotational inertia in the others. Generally, the size, geographical location and the number of areas with these characteristics is arbitrary, but all of them should be synchronized to each other. The size of the system could vary from a small region or a larger synchronous area in the continental Europe.

In [4], authors demonstrate that in a network with uneven distribution of inertia, there would be a big difference between the frequency of the system at the Center of Inertia (COI) and the frequency at low inertia regions immediately after the disturbance. A study on the transient power flows over the tieline between synchronizing areas is proposed in [2]. It is shown that the overshoot, magnitude and the oscillation frequency will be increased (by more than 50\%) and becoming more abrupt (by up to $300 \%$ ) in case of uneven distribution of inertia in two interconnected and synchronized areas. In a grid situation as described here, a false short circuit event may be detected by the protection devices leading to the immediate false tripping of the tie-line in an already sensible moment and cascading tripping. The results proposed in [5] show that generators electrically closest to the disturbance are influenced the most and if this generator(s) has relatively low inertia, it could see a local RoCoF many times greater than the system RoCoF. It was also observed that when a large portion of the total power system inertia is concentrated at one machine, the mean of the local RoCoF is significantly larger compared to when the power system inertia is equally distributed across all machines. In [6-8] authors propose the effect of uneven inertia distribution on local RoCoF, system small signal stability and transient stability on the ERCOT power system. They claimed that inertia contributes more to local frequency stability than the global (system) stability. In addition, the local inertia reduction significantly increases the local oscillations during the incidents close to these areas. Due to decreased local inertia, some system modes oscillate faster and their damping ratio tends to the negative values, which causes an unstable system condition.

A comprehensive report from Australian Energy Market Operator (AEMO) for the blackout at the South Australia clearly indicates that one of the main reasons for such a blackout is the lack of inertia in the southern area of the system, while the northern area operated with greater inertia. This uneven distribution of inertia resulted in a substantially faster RoCoF compared to the other events, exceeding the ability of the under frequency load shedding schemes to arrest the frequency fall before it dropped below 47Hz [9]. AEMO is currently pursuing a project titled as "Future Power System Security Program (FPSSP)" which takes a strategic approach to studying the security requirements of future power systems, especially frequency control and RoCoF following a change

This work was supported by Elia, Belgium's electricity transmission system operator and received the support of Energy Transition Funds (SPF Economy) 
in inertial response [10]. In addition, in one part of the PanEuropean Transmission System, (e-Highway 2050)" project [11] a dynamic simulation focused on the frequency stability of the future European power system demonstrates the effect of local inertia phenomenon through a larger synchronous area [12].

Based on this and in order to identify and analysing the possible risks due to the local inertia phenomenon in detail, the Belgium's electricity transmission system operator (Elia), funded an industrial research project in collaboration with the Brunel University London to study such phenomenon in Belgian power system and possible mitigation measures. Consequently, Elia intends to further investigate the possible transposition or extrapolation of such effects on a larger synchronous area with a focus on possible local consequences in Belgium due to an uneven inertia distribution in continental Europe.

In the first phase of the project, Elia intends to start by understanding the physics behind the possible effects of the uneven distribution of inertia. The final objective of this phase is to understand if local (i.e. Belgian) phenomenon or the altered behaviours of the neighbours in the continental Europe can impact the stability of the Elia grid and then trigger the need for mitigation measures. This paper presents the results obtained from the Step-1 of the first phase of this project based on the outcomes of simulations and studies.

In this task, the project partners also take the advantage of collaborating with the UK National Grid (NG) which is currently reviewing the results of a series of funded projects related to the inertia in the GB power network. In "Enhanced Frequency Control Capability (EFCC)" project, NG is aiming to provide greater clarity on the application and benefits of innovative ways to control the system frequency in a low inertia situation [13]. In addition, NG is currently funds a research project as " $(F 2 P)$ " to understand the effect of fast frequency phenomena in the GB power system [14]. Results of the preliminary studies showed that a low inertia condition close to the event stimulates this issue.

Due to some events in the GB power system relating to the local inertia phenomenon, large inertia difference between the Scotland and England grid and islanding operation, the GB power system is adopted at the starting point of the study. A reduced model of this system already exists and is publicly available. In this project this model is used for simulations, but at first it is verified with real measurements provided by NG.

This paper is organized as follows. In section II, the concept of local inertia phenomenon is described. Then in sections III and IV, the GB transmission system reduced model is introduced and validated respectively. Finally, in section $\mathrm{V}$, relevant effects of local inertia phenomenon is investigated and discussed.

\section{OVERALL AND DISTRIBUTION SYSTEM INERTIA}

The well-known classical swing equation for a single generator with the nominal power of $S_{n}$ and inertia constant $H$ defines the relation between the RoCoF, $\dot{f}_{m}$ and the power imbalance as,

$$
\dot{f}_{m}=-\Delta P \frac{f_{0}}{2 H S_{n}}
$$

where $\Delta P=P_{m}-P_{e}$ is the difference between mechanical input power $P_{m}$ and electrical output power $P_{e}$ in pu values. Equation (1) shows that a power imbalance in turbinegenerator results in the frequency derivative. According to (1), the value of RoCoF depends to the quantity of power imbalance and inertia of the generator. However, real power systems consist of a large number of generators and when an imbalance in the system arises, frequency is not uniform throughout the system. In this case, a common method used to predict the initial system frequency deviation after a disturbance is by extending the Equation (1) as,

$$
\dot{f}_{s y s}=-\Delta P_{s y s} \frac{f_{0}}{2 H_{s y s} S_{n, s y s}}
$$

where $S_{n, s y s}=\sum_{i=1}^{N} S_{n i}, S_{n i}$ is the rated power of generator $i$ [MVA]. $\Delta P_{s y s}=\sum_{i=1}^{N}\left(P_{m}^{i}-P_{e}^{i}\right)$ and the value of $H_{s y s}$ denotes to the total inertia constant of the system and can be obtained from:

$$
H_{s y s}=\frac{\sum_{i=1}^{N} S_{n i} H_{i}}{S_{n, s y s}}
$$

This method assumes that the power system can be considered as a single machine, possessing the total power system inertia connected to a single bus with a single system frequency and RoCoF. However, the results in real power systems reveal that this assumption is possibly too simplistic and unreal, as this method does not take account of the local oscillations at the individual generators since the local generators respond with different rates in case of system disturbance.

Figure 1 demonstrates the effect of the local inertia phenomenon on the generators speed deviations of the IEEE 39-bus test system. In Figure 1(a), the inertia is distributed equally across the system while in Figure 1(b) most of the system inertia is concentrated in generator G01 at the point of the interconnection to the rest of the system. In both cases the system total inertia is kept constant and a similar generation loss event including the tripping of G09 is applied. As can be seen, for equal distribution of inertia in Figure 1(a), the speed deviation at the COI and at the local generators are close to each other. However, when the system inertia are mostly concentrated at one point i.e., G01, there are some large speed deviations in different generators of the system especially for those near to the tripped generator/event. This is despite the fact that the whole system inertia remains constant and the COI speed variations is almost similar. These deviations in speed can influence the system frequency, RoCoF and generators stability in some cases, depending on the size and configuration of the uneven inertia distribution in the system.

\section{REDUCED MODEL OF GB TRANSMISSION SYSTEM}

A 36-zone equivalent network representing the GB transmission system is first developed by NG in 2012 using Power-Factory. The entire GB transmission system is split into 36 zones for which the relevant generation and demand are aggregated. Each zone demonstrates a geographical area of the system. Generators within each zone are categorised into fuel types and the demand was split into active and reactive parts. Line impedances were also calculated to represent the electrical distance between the zones. The single 
line diagram and structure of each zone are illustrated in Figures 2 and 3 respectively.

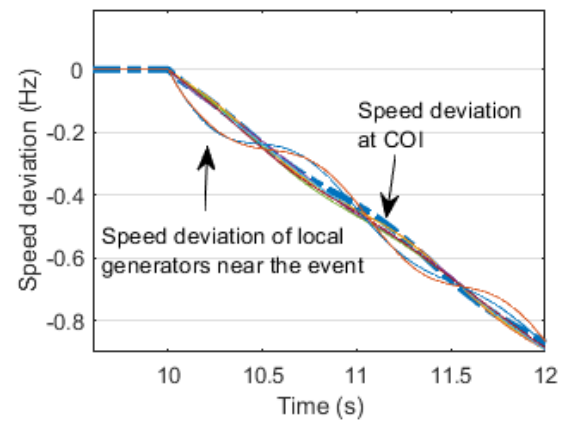

(a)

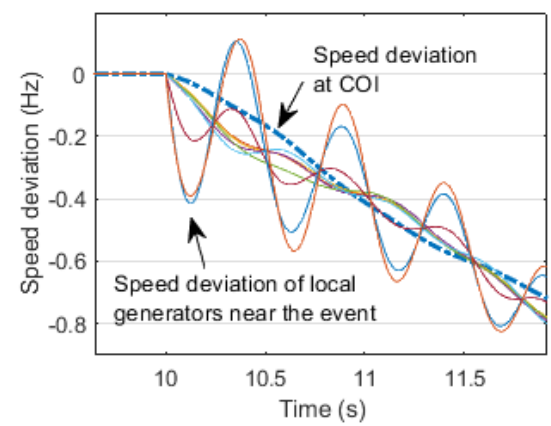

(b)

Figure 1 Local and COI Speed deviation in case of (a) equal inertia distribution (b) uneven inertia distribution

In this study, this model is updated and validated to the prevalent system condition to give a confidence to the results. For this approach, NG supported the authors with data relating to the system configuration as well as real recorded data for a 500MW HVDC link $2 a$ trip and 630MW nuclear power plant trip. Updating and validating the model is performed with the following steps,

\section{A. Generation}

Generation configuration including conventional generation, HVDC interconnectors and RES is updated to the existing GB transmission system up to the 2018 system. The inertia of synchronous generators is updated according to the relevant units aggregated at each zone, by using (3).

\section{B. Load modelling}

The simplified exponential load model contains voltage and frequency dependent terms is:

$$
\begin{gathered}
P=P_{n}\left(\frac{V}{V_{n}}\right)^{K_{P V}}\left(1+K_{P F} \Delta f\right) \\
Q=Q_{n}\left(\frac{V}{V_{n}}\right)^{K_{Q V}}\left(1+K_{Q F} \Delta f\right)
\end{gathered}
$$

where $P$ and $Q$ denote to real and reactive power, respectively, at voltage $V$ and frequency $f, P_{n}$ and $Q_{n}$ are real and reactive power at rated voltage $V_{n}$ and rated frequency $f_{n}$, $K_{P V}$ and $K_{Q V}$ are real and reactive power voltage exponents and $K_{P F}$ and $K_{Q F}$ are real and reactive power frequency exponents [15]. While at the time of the events, the system mostly supplied with the lighting and heating loads, typical parameters for combined lighting and heating were used for modelling the load. The following parameters are adopted as: $K_{P V}=0.96, K_{Q V}=7.4, K_{P F}=1$ and $K_{Q F}=-2.8$.

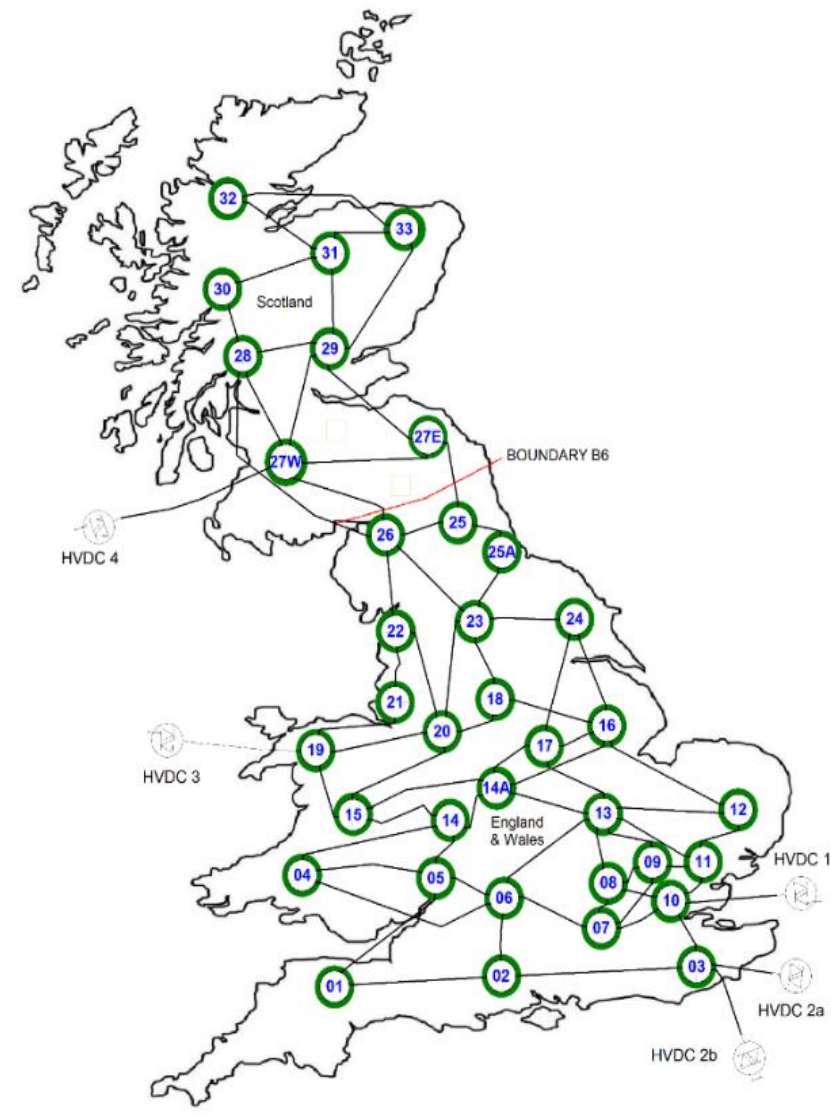

Figure 2 Single line diagram of 36-Zone GB transmission network

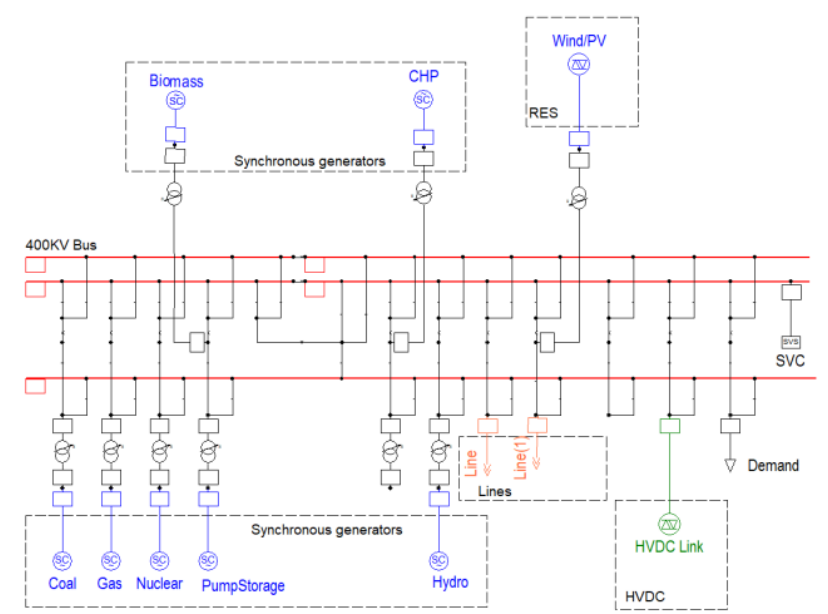

Figure 3 Structure of each zone

\section{Validating the model}

A scenario is created with the similar configuration to that of the actual system at the time of the event, same generation, demand and inertia distribution in each zone. System frequency at six points, i.e. Zones 01, 03, 18, 27E, 29 and 32 are compared with real quantities recorded by PMU. Figure 4 shows the simulation results for the system frequency at zone 03 and the associated real ones recorded by a PMU at this point. As can be seen, in the PMU measurement the frequency Nadir is around $49.74 \mathrm{~Hz}$. For the simulation however, the initial slope of the frequency reduction is similar to the measurement, but there is a significant difference on frequency Nadir (about $0.17 \mathrm{~Hz}$ ). The same slope in frequency 
reduction suggests that the simulated inertia in the model is close to the actual value, while the difference in the frequency Nadir indicates the requirement of fine-tuning the parameters of the generators controller blocks especially the governors.

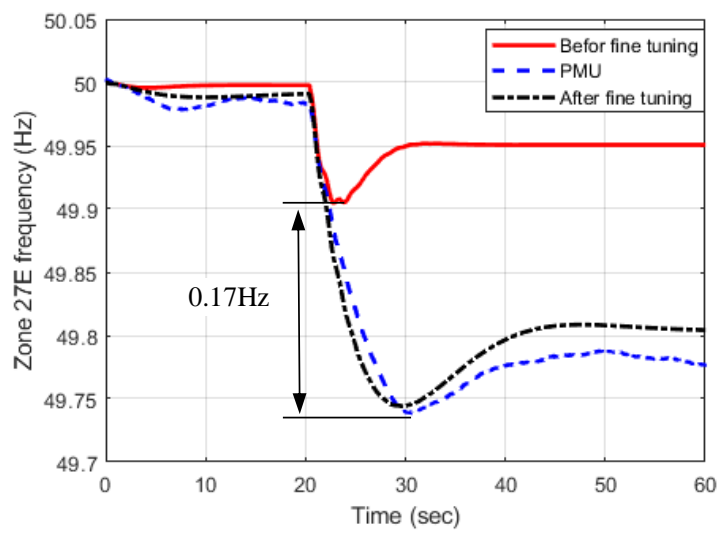

Figure 4 Frequency at Zone 03 before and after tuning the model

\section{Fine-tuning}

After detailed investigations and looking at the swing equation, i.e. Eq. (1), there are two main parameters affecting the frequency: inertia $(H)$ and mechanical input $\left(P_{m}\right)$. As stated in the previous section and inferred from Figure 4 , the inertia simulated in the model is close to the real system since the slope of the frequency reduction for both simulation and measurement immediately after the event are similar. For tuning the model we focus on another parameter, $P_{m}$ which is the turbine mechanical output power and is mostly determined by the response of the turbine governor.

Power-Factory implements the following model for simulating the control block for synchronous generators as shown in Figure 5. In this model, the combination of Primary Controller (PCO) and Prime Mover Unit (PMU) constructing the governor block as illustrated in Figure 6 for a standard TGOV1 model. The time constants of $T_{1}, T_{2}$ and $T_{3}$ are the time constant associated with the motion of steam through reheater and turbine stages. $D_{t}$ is turbine damping coefficient and $R$ is turbine-governor droop. $V_{\min }$ and $V_{\max }$ are main steam control valve minimum and maximum limit respectively. The time constants of the original model was adjusted to obtain more realistic results. By this, the response of the governors becomes slower and the response of the system inertia could be revealed. After this adjustment, as depicted in Figure 4, the results from simulations and real measurements are mostly identical.

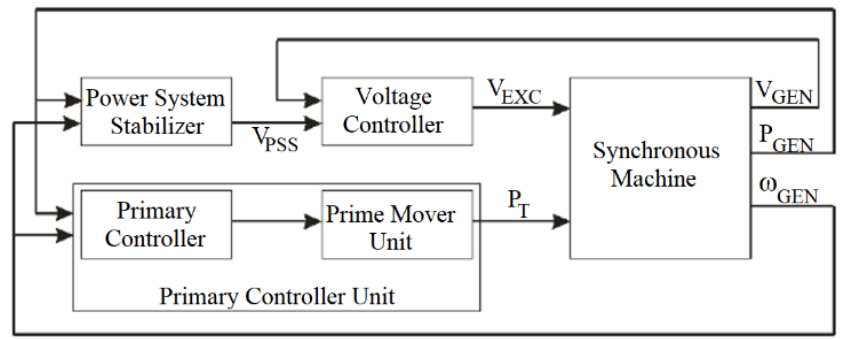

Figure 5 Power-Factory model for control block of synchronous generators

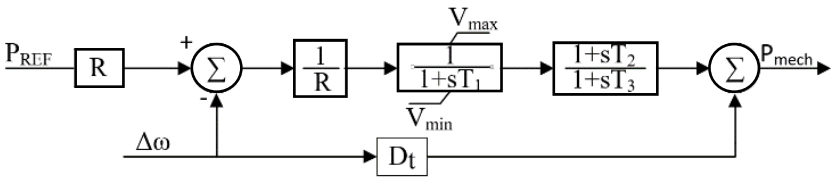

Figure 6 Block diagram of turbine governor

\section{LOCAL INERTIA ON GB TRANSMISSION SYSTEM}

In this section, the concept of uneven distribution of inertia across GB synchronous power system is studied and how that can be related to the concepts such as local inertia and regional inertia is investigated and discussed. The base case for this study is the verified reduced model of the GB transmission system described in the previous sections. This section tries to demonstrate the concept and impact of uneven inertia distribution across a synchronous power system, through simulating some relevant events.

The GB reduced transmission system single line diagram as well as its inertia distribution is shown in Figure 7. The GB transmission system is represented in two main regions, the southern part includes England and Wales as indicated by R1 region and Scotland in the north indicated by $\mathrm{R} 2$ region. For this case, the whole network was operated in a synchronized condition while there was a $1200 \mathrm{MW}$ export from R2 to the $\mathrm{R} 1$ through two embedded transmission lines as depicted on Figure 7 by thick black lines. The distribution of inertia are clearly indicated on this figure by different colouring theme. Zones with purple and red denote to high inertia regions incorporating large synchronous generations, blue zones have an average inertia and green zones with relatively low inertia and more wind generations. There is no synchronous generation in zones without colouring, so it is considered that these zones have zero rotational inertia. The tie-lines connecting the region $\mathrm{R} 1$ and $\mathrm{R} 2$ are loosely transmission lines with significant length $(100 \mathrm{~km}$ and $50 \mathrm{~km}$ for $26-27 \mathrm{~W}$ and $25-27 \mathrm{E}$ respectively). The network stability in $\mathrm{R} 2$ is very sensitive to the operation of these lines.

While there exists large in-service synchronous (coal, gas and nuclear) power-plants at Zones 04, 06, 16, 17, 22, 23 and 24 in the R1, most generations in R2 is from non-synchronous (wind) and small hydro units. Consequently, the R1 occupies almost the $89.59 \%$ of the total system inertia with strong interconnections between zones compared with the R2 with just about $10.41 \%$ of the total system inertia and weaker transmission system. As seen, the total inertia in the R1 (England and Wales) is about nine times greater than the R2 (Scotland) for this dispatch. Since these regions are synchronized but connected through transmission lines, it can be inferred that the inertia distribution for these two synchronous regions is not uniformly distributed and the R2 could be interpreted as local inertia in compare to the total power network or R1. In other words, from R2 point of view, the $\mathrm{R} 1$ could be modelled as a large synchronous machine with a great inertia connected by two weak transmission lines to the R2.

\section{EFFECT OF UNEVEN INERTIA DISTRIBUTION}

After studying the results of the extensive simulations that is performed in different scenarios, the effect of local inertia phenomenon may be summarized into three main issues: 1) higher values in local RoCoF, 2) increasing in the magnitude and frequency of inter-area oscillations and 3) changing in the 
Critical Clearing Time (CCT) of embedded transmission lines. The last two issues also can be linked to the system small signal and transient stability respectively. Following section describe theses effects on GB transmission system.

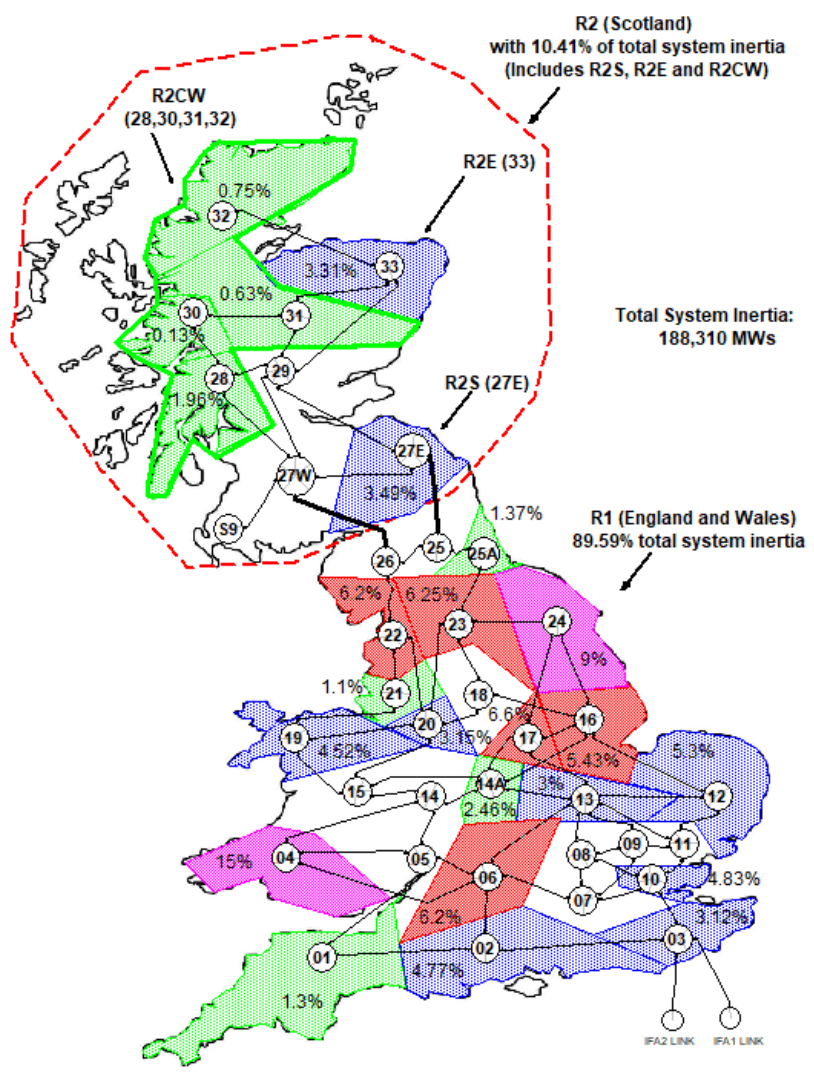

Figure 7 GB reduced model with inertia distribution

\section{A. Local RoCoF}

The RoCoF in R2 is monitored for the same size of generation loss (400MW) in different locations, i.e. in zones 24 (R1), 27E (R2S), 28 and 32 (R2CW) with different inertia distribution in the system. The box and whisker plot in Figure 8 demonstrates the RoCoF in two cases: case 1 with base inertia distribution as depicted in Figure 7 and case 2 with 50\% reduction of inertia in $\mathrm{R} 2$ while the system total inertia is kept constant. For this purpose, the equivalent value of the reduced inertia in $\mathrm{R} 2$ is added to R1.

RoCoF measurement is calculated for the frequency deviation of the first 500ms after the event. Results in Figure 8 indicate an increase in $\mathrm{RoCoF}$ by increasing the distance from the inertia concentration centre (R1). This is clear from Figure 8 as the RoCoF values for the generation loss at zones $27 \mathrm{E}, 28$ and 32 are significantly larger than those values for generation loss at zone 24 which is located at the inertia concentration center. In addition, in case 2 for more uneven inertia distribution in the system, the value of the most probable of highest RoCoFs (the length between upper and lower whiskers), its variability (the height of the box and its side distance from the median as indicated by a horizontal red line) and its extreme values (+ signs), are significantly increased compared to the case 1 [17]. This study demonstrates the effect of local inertia on the local RoCoF as with more uneven inertia distribution, the value of the local RoCoF is increased.

\section{B. Inter-Area oscillations}

In this case, the active power oscillation in the tie-line 25$27 \mathrm{E}$ in case of significant generation loss in R2 (630MW generation trip in Zone 27E) is simulated and studied. The inertia of R 2 region is decreased by $50 \%$ and $75 \%$ steps and added to R1 so that the total inertia of the system stays constant.

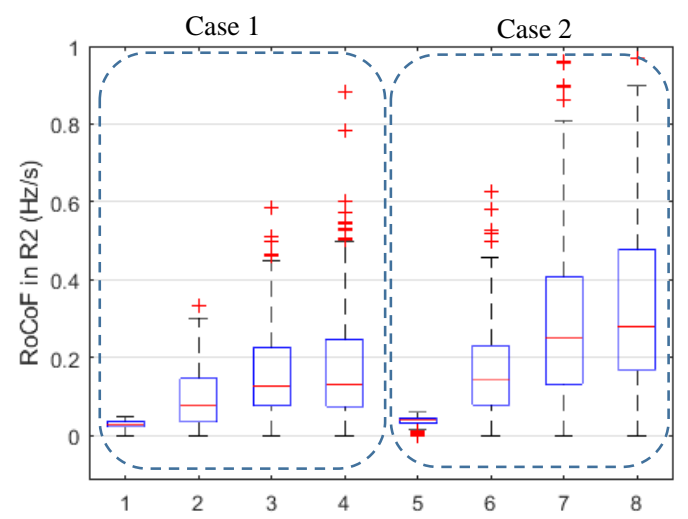

Figure 8 Effect of inertia distribution on local RoCoF

1 4: Generation trip at 24, 27E, 28 and 32 respectively (case 1)

5 8: Generation trip at 24, 27E, 28 and 32 respectively (case 2)

Results are plotted in Figure 9. The increase in both overshoot and damping frequency $\left(\omega_{d}\right)$ of the tie-line active power in case of inertia reduction in $\mathrm{R} 2$ is inevitable. The preliminary results indicate that as long as the system is stable, the damping ratio $(\zeta)$ also increases as a result of reduction in $\mathrm{R} 2$ inertia. It can be inferred that the uneven distribution of inertia has a sensible impact on inter-area oscillations overshoot, damped natural frequency and damping ratio.

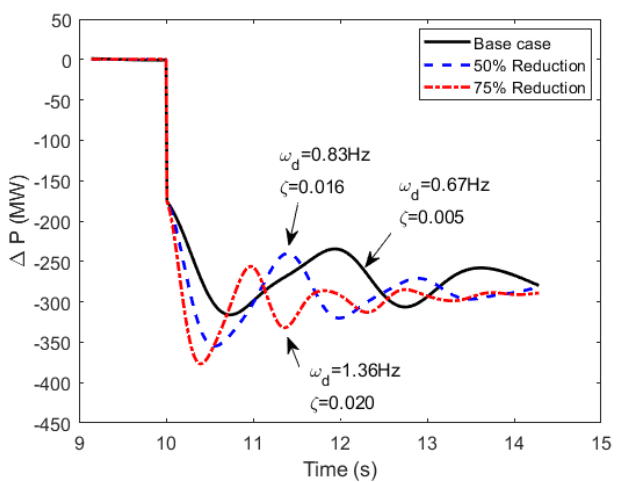

Figure 9 Tie-lines 25-27E active power oscillations with changes in R2 inertia

\section{Critical Clearing Time (CCT)}

A three phase fault applied to the line 25-27E in Figure 7 with the duration of $120 \mathrm{~ms}$. Table I shows the CCT of this line for different inertia distribution in R2. The CCT value for the base case is $294 \mathrm{~ms}$ and it is reduced to $263 \mathrm{~ms}$ when the total inertia of R2 is reduced up to $50 \%$ in case 1 . In cases 2 and 3 the total inertia of R2 is constant but is distributed differently in R2S and R2E. As seen, for greater inertia in R2S the CCT is at its maximum value of $305 \mathrm{~ms}$ while this value is $269 \mathrm{~ms}$ for case 3 where the inertia of R2E is increased. This indicates that the regions with higher inertia are more capable to absorb and prevent the fault to be spreading in the system when they are close to the fault and finally better system transient stability for those faults. In other words, in a system with uneven inertia distribution, the regions with a higher inertia have a better transient stability for close faults. Figure 10 
shows the active power oscillations of another tie-line (26$27 \mathrm{~W}$ ) in case of inertia reduction in $\mathrm{R} 2$ with the same disturbance. As can be seen, the magnitude and the frequency of the power oscillations in this healthy line are increased significantly which can be a threat for false detection of protection schemes of this line and complete islanding of two regions (England and Scotland).

TABLE I. CCT OF LINE 25-27E IN DIFFERENT INERTIA DISTRIBUTION

\begin{tabular}{ccc}
\hline Case & Inertia distribution scheme & CCT (ms) \\
\hline Base case & --- & 294 \\
Case 1 & Reducing 50\% from R2 and adding to R1 & 263 \\
Case 2 & Reducing 50\% from R2CW and adding to R2S & 305 \\
Case 3 & Reducing 50\% from R2CW and adding to R2E & 269 \\
\hline
\end{tabular}

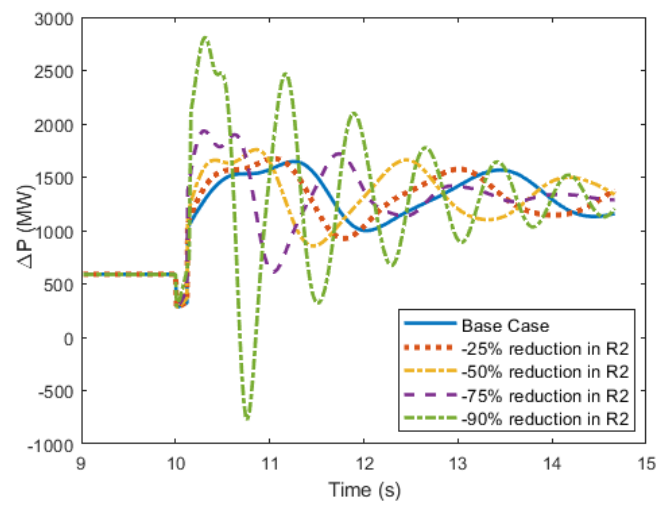

Figure 10 Tie-lines active power oscillations in case of different inertia distribution

\section{Frequency stability}

In this section the effect of inertia distribution on system frequency stability is simulated and studied. For this, a generation loss equal to $1800 \mathrm{MW}$ (the reference incident in GB [16]), is simulated in Zone 32. Figure 11 shows the frequency of the COI and R2 when $90 \%$ of $\mathrm{R} 2$ inertia is reduced and transferred to the $\mathrm{R} 1$ (the total inertia of system is kept constant).

As can be seen, not a significant change observed for the frequency at the COI in two cases since the COI frequency is a parameter of system total inertia that is constant. Despite of the COI frequency and frequency Nadir, the local frequency oscillations increase significantly (in Zones 27E, 32 and 33), when the inertia of $\mathrm{R} 2$ is reduced. It can be inferred that the local frequency oscillations are more affected from uneven distribution of inertia however; the frequency at COI and its minimum value is more affected from the total system inertia.

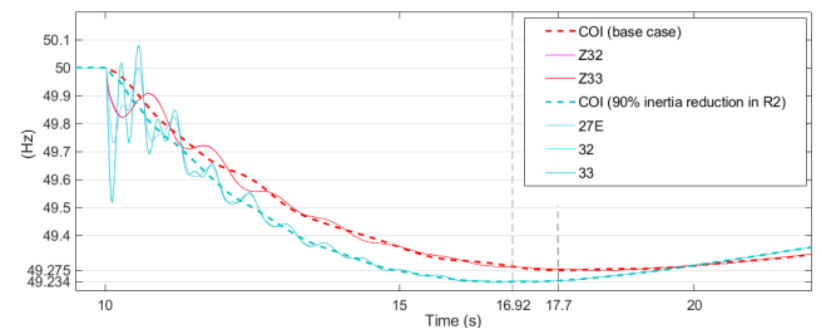

Figure 11 Effect of inertia distribution on COI frequency

\section{CONCLUSION}

The concept of local inertia phenomenon is introduced and discussed by simulating some relevant cases on GB reduced model. It was shown that the GB power system could be a good reference for studying the local inertia phenomenon, as there is a significant inertia distribution difference between England and Wales with Scotland. It was shown that the concept of the local inertia phenomenon is directly associated with a region with low inertia which is synchronized to a high inertia region. The results of simulations generally demonstrate the effect of local inertia as, increasing the system local RoCoF, more vulnerability to the small signal instability, greater inter-area oscillations and smaller critical clearing time of transmission lines regarding the system transient stability. For the future works, the effect of various parameters (such as load inertia, generation dispatch, and network configuration) should be investigated and studied.

\section{ACKNOWLEDGMENT}

All supports and guidance from UK National Grid for providing the GB power system data, snapshots and real measurements is highly appreciated.

\section{REFERENCES}

[1] "Technical and economic analysis of the European electricity system with $60 \%$ RES", EDF research and development division, June 2015 ,

[2] A. Ulbig, T. S. Borsche and G. Andersson, "Impact of Low Rotational Inertia on Power System Stability and Operation", Proceedings of the $19^{\text {th }}$ World Congress, The International Federation of Automatic Control Cape Town, vol. 47, no. 3, pp. 7290-7297, South Africa, 2014

[3] "Frequency Stability Evaluation Criteria for the Synchronous Zone of Continental Europe", ENTSO-E, March 2016,

[4] P. Wall, N. Shams, V. Terzija, et al., "Smart frequency control for the future GB power system", IEEE PES Innovative Smart Grid Technologies Conference Europe (ISGT-Europe), 2016

[5] D. Doheny and M. Conlon, "Investigation into the local nature of rate of change of frequency in electrical power systems", $52^{\text {nd }}$ International Universities Power Engineering Conference (UPEC), 2017

[6] T. Xu, W. Jang and T. J. Overbye, "Investigation of inertia's locational impacts on primary frequency response using large-scale synthetic network models", IEEE Power and Energy Conference at Illinois (PECI), 2017

[7] T. Xu, W. Jang and T. J. Overbye, "Location-Dependent Impacts of Resource Inertia on Power System Oscillations", Proceedings of the $51^{\text {st }}$ Hawaii International Conference on System Sciences, 2018

[8] T. Xu, Y. Liu and T. J. Overbye, "Metric Development for Evaluating Inertia's Locational Impacts on System Primary Frequency Response", IEEE Texas Power and Energy Conference (TPEC), 2018

[9] "Black System South Australia 28 September 2016" report, Australian Energy Market Operator (AEMO), March 2017

[10] "Future Power System Security Program", Progress Report, August 2016

[11] "Europe's future secure and sustainable electricity infrastructure", $e$ Highway 2050 project results, November 2015,

[12] A. Kasembe, K. Máslo, S. Moroni and et al. "Frequency stability of the future continental Europe power system", Proceedings of the $18^{\text {th }}$ Mediterranean Electro-technical Conference, MELECON, 2016

[13] "Enhanced Frequency Control Capability (EFCC)", Progress report: January to June 2017 ,

[14] F2P - Investigation \& Modelling of Fast Frequency Phenomena, National grid and Brunel University London, Progress report, 2018,

[15] L. M. Korunovi, J. V. Milanovi, S. Z. Djokic, et al., "Recommended Parameter Values and Ranges of Most Frequently Used Static Load Models", IEEE Trans. on Power Systems, vol. 33, no. 6, pp. 5923-5934, 2018

[16] "Voltage and Frequency Dependency", System Operability Framework, National Grid, UK, 2018,

[17] "boxplot" help, MATLAB R2019a, document is available online at: https://uk.mathworks.com/help/stats/boxplot.html 